\title{
Comparison of perinatal outcomes in facilities before and after Global Network's Helping Babies Breathe Implementation Study in Nagpur, India
}

Archana Patel ${ }^{1}$, Akash Bang ${ }^{2 *}$, Kunal Kurhe ${ }^{1}$, Savita Bhargav ${ }^{1}$, Amber Prakash${ }^{1}$, Spoorthy Arramraj ${ }^{3}$ and Patricia L. Hibberd ${ }^{4}$

\begin{abstract}
Background: The Helping Babies Breathe (HBB) educational program focuses on training of first-level birth attendants in neonatal resuscitation skills for the first minute of life (The Golden Minute). Pre-post studies of HBB implementation in sub-Saharan Africa and Asia have shown reductions in facility-based very early neonatal mortality and stillbirth rates. However, the Global Network pre-post HBB Implementation Study (GN-HBB-IS) found no difference in day 7 perinatal mortality rates (PMR-D7) among births to women participating in the Global Network's Maternal and Newborn Health Registry. To address potential differences in perinatal outcomes in births occurring in facilities that implemented HBB vs. all births occurring in the communities served by facilities that implemented $\mathrm{HBB}$, we compared day-1 perinatal mortality rates (PMR-D1) among births occurring pre and post HBB implementation in facilities in Nagpur, India, one of the 3 sites participating in the GN-HBB-IS.
\end{abstract}

Methods: We hypothesized that there would be a 20\% decrease in the Nagpur facility based PMR-D1 in the 12 months post GN HBB implementation from the pre-period. We explored pre-post differences in stillbirth rates (SBR) and day-1 neonatal mortality rates (NMR-D1).

Results: Of the 15 facilities trained for the GN-HBB-IS, 13 participated in the Nagpur HBB Facility Study (NagpurHBB-FS). There were 38,078 facility births in the 12 months before the GN-HBB-IS and 40,870 facility births in the 12 months after the GN-HBB-IS. There was 11\% overlap between the registry births analyzed in the GN-HBB-IS and the facility births analyzed in the Nagpur-HBB-FS. In the Nagpur-HBB-FS, there was a pre-post reduction of $16 \%$ in PMR-D1 $(p=0.0001)$, a $14 \%$ reduction in SBR $(p=0.002)$ and a 20\% reduction NMR-D1 $(p=0.006)$.

Conclusions: In the Nagpur-HBB-FS, PMR-D1, stillbirths and NMR-D1 were significantly lower after HBB implementation. These benefits did not translate to improvements in PMR-D7 in communities served by these facilities, possibly because facilities in which HBB was implemented covered an insufficient proportion of community births or because additional interventions are needed after day 1 of life. Further studies are needed to determine how to translate facility-based improvements in PMR-D1 to improved neonatal survival in the community.

Trial registration: The Global Network HBB Implementation Study (GN-HBB-IS) was registered at ClinicalTrials.gov: NCT01681017.

Keywords: Helping babies breathe, Asphyxia, Neonatal mortality, Perinatal mortality, Stillbirths, Essential newborn care, Training, Pre-post design, Facility births, Community births, Implementation science, Global Health, India

\footnotetext{
* Correspondence: drakashbang@gmail.com

${ }^{2}$ Mahatma Gandhi Institute of Medical Sciences, Sewagram, Maharashtra

State 442102, India

Full list of author information is available at the end of the article
}

(c) The Author(s). 2019 Open Access This article is distributed under the terms of the Creative Commons Attribution 4.0 International License (http://creativecommons.org/licenses/by/4.0/), which permits unrestricted use, distribution, and reproduction in any medium, provided you give appropriate credit to the original author(s) and the source, provide a link to the Creative Commons license, and indicate if changes were made. The Creative Commons Public Domain Dedication waiver (http://creativecommons.org/publicdomain/zero/1.0/) applies to the data made available in this article, unless otherwise stated. 


\section{Background}

Neonatal deaths now account for around $46 \%$ of the under-5-years-old deaths and must be addressed to accelerate progress towards Sustainable Development Goal 3 (SDG3) [1-4], because one of the targets under SDG3 is to reduce the neonatal mortality to 12 per 1000 livebirths by 2030 [5]. While neonatal deaths have fallen from 5 to 2.5 million from 1990 to 2017, the annual rate of reduction in neonatal mortality over this period (2.6\% per year) is much lower than that for children aged 1-59 months (3.7\%) [1]. Among live-born babies, the risk of death is greatest on the first day of life-about 1 million deaths a year and $36 \%$ of all neonatal deaths [2]. Many of these deaths are caused by birth asphyxia or failure to establish breathing at birth. Excluded from these deaths are the estimated 1.3 million intrapartum or "fresh" stillbirths [6] who are not breathing at birth and for some reasons, are subjected to either inadequate or no resuscitation at all. For all these reasons, there is an intense global focus on interventions for the estimated 10 million babies requiring assistance to initiate breathing $[7,8]$.

Around $85 \%$ of babies born at term initiate spontaneous respiration within 10 to $30 \mathrm{~s}$ of birth, an additional $10 \%$ require initial steps such as tactile stimulation or airway clearing or positioning [9-13] and approximately $3 \%$ require positive-pressure ventilation by bag and mask [11, 13-15]. Training in these basic resuscitation measures should be able to salvage $90 \%$ of the babies that do not initiate spontaneous respiration and is most needed in low resource settings where there is inadequate access to intrapartum and immediate post-partum care [13, 16, 17]. Helping Babies Breathe (HBB) is a simple hands-on training curriculum in basic newborn resuscitation for birth attendants [18]. The training focuses on appropriate resuscitation skills to be used within the first $60 \mathrm{~s}$ of life (the Golden Minute) including timely delivery of the essential interventions such as drying, providing warmth, and clearing the airway, providing additional stimulation to breathe and bag and mask ventilation, if needed. In past, studies have attempted to quantify the impact of resuscitation on newborn outcome and have shown the benefits of resuscitation training on newborn outcomes, including the Bang et al. study in which resuscitation training in India reduced the asphyxia-related mortality by $65 \%(p<0.02$, [19]). Carlo et al. used a "train-the-trainer" model to sequentially train midwives in urban, community health clinics in Zambia in Essential Newborn Care (ENC) and in the American Academy of Pediatrics Neonatal Resuscitation Program (NRP), and found a decrease in the all-cause 7-day neonatal mortality rates from 11.5 to 6.8 deaths per 1000 live births after ENC training, which was further lowered by NRP training. Recently the Eunice Kennedy Shriver National Institute of Child Health and Human Development (NICHD) Global Network for Women's and Children's Health Research, and
Research Triangle International (RTI) as the data coordinating center, conducted a pre-post study to evaluate the impact of $\mathrm{HBB}$ implementation in facilities serving rural communities (Maternal and Newborn Health Registry (MNHR)) located at three Global Network research sites - Nagpur and Belgaum in India and Eldoret in Kenya [18, 20]. MNHR enrolls pregnant women and records maternal and neonatal outcomes in catchment areas of rural primary health centers (study clusters). An additional file shows the flow diagram for the Nagpur Site of the Global Network HBB Implementation Study (GNHBB-IS) [See Additional file 1]. All the births in the community based $\mathrm{MNH}$ registry were analyzed in the GN-HBB-IS. Around 45\% of these did deliver in HBB trained facilities but rest delivered outside in other facilities. GN-HBB-IS did not find any effect of HBB implementation on day 7 perinatal mortality (PMR-D7). There are two plausible reasons for the failure to observe a reduction in PMR-D7 after facility-based health workers were trained in HBB implementation. Firstly, the reduction in PMR-D7 was estimated in all deliveries of pregnant women enrolled from the communities served by the trained facilities, not just those who delivered in $\mathrm{HBB}$ trained facilities. Secondly, the pre training period overlapped with the period of HBB training that was carried out in a step wise manner across the facilities and could have already started to reduce PMR-D7 in the pre-HBB implementation period (Table 1 and Additional file 1).

Since the GN-HBB-IS was not designed to evaluate facility based changes in day 1 perinatal mortality (PMR-D1), we planned this facility based study (Nagpur-HBB-FS) and collected data from the Nagpur facilities participating in GN-HBB-IS before any training had commenced and after the GN-HBB-IS training had been completed for all the births in the facilities irrespective of whether or not they belonged to the MNH registry. Our objective was to evaluate PMR-D1 in births occurring in facilities pre-compared to post- GN-HBB-IS implementation.

\section{Methods}

Study site for the facility based study

This study (The Nagpur-HBB-FS) was conducted and coordinated by the research foundation located at Nagpur, which is a Global Network site [20]. In this study we included 13 of the 15 facilities that had participated in the GN-HBB-IS as two tertiary level facilities declined consent for sharing their pre-training data. The 13 facilities that participated in the Nagpur-HBB-FS included 2 primary facilities (facilities where caesarian section facility is not available), 4 secondary facilities (where caesarian section facility is available on call) and 7 tertiary facilities (where caesarian section facility is available round the clock along with allied emergency services). All the facilities except the tertiary facilities were located in rural 
Table 1 Comparison between the Nagpur-HBB-FS and the Nagpur Site of the GN-HBB-IS

\begin{tabular}{|c|c|c|}
\hline Criteria & $\begin{array}{l}\text { Nagpur HBB Facility Study } \\
\text { (Nagpur-HBB-FS) }\end{array}$ & $\begin{array}{l}\text { Nagpur Site of the GN HBB Implementation } \\
\text { Study (GN-HBB-IS - Nagpur) }\end{array}$ \\
\hline Study Population & $\begin{array}{l}\text { - All births in } 13 \text { of the } 15 \text { facilities that had } \\
\text { participated in the GN-HBB-IS whether } \\
\text { they belonged to the MNH registry area } \\
\text { (around } 11 \% \text { ) or not. }\end{array}$ & $\begin{array}{l}\text { - All births in the GN MNH Registry whether } \\
\text { delivered at facilities that participated in the } \\
\text { GN-HBB-IS (around 45\%) or at other facilities } \\
\text { that did not receive HBB training and } \\
\text { implementation. }\end{array}$ \\
\hline Inclusion Criteria & - All stillbirths included & - Only fresh stillbirths included \\
\hline Exclusion Criteria & $\begin{array}{l}\text { - Miscarriage } \\
\text { - Medical Termination of Pregnancy (MTP) }\end{array}$ & $\begin{array}{l}\text { - Miscarriage } \\
\text { - Medical Termination of Pregnancy (MTP) } \\
\text { - Birth weight }<1500 \mathrm{~g} \\
\text { - Missing birth weight } \\
\text { - Macerated stillbirths }\end{array}$ \\
\hline Facility HBB Training Period & June 2012 to October 2012 & \\
\hline Pre HBB data collection Timing & - April 2011 - March 2012 & - November 2011-October 2012 \\
\hline Post HBB data collection Timing & - November 2012-October 2013 & • November 2012-October 2013 \\
\hline Outcomes & $\begin{array}{l}\text { - PMR-D1 - (All stillbirths + day } 1 \text { neonatal mortality) } \\
\text { - All stillbirths } \\
\text { - NMR-D1 (Day } 1 \text { neonatal mortality) }\end{array}$ & $\begin{array}{l}\text { - PMR-D7 (only fresh } \\
\text { stillbirths + day } 7 \text { neonatal mortality) } \\
\text { - Only fresh stillbirths } \\
\text { - PMR-D1 (only fresh stillbirths + day } 1 \\
\text { neonatal mortality) }\end{array}$ \\
\hline Data Source & $\begin{array}{l}\text { - Pre-existing standard facility records like birth } \\
\text { and mortality registers }\end{array}$ & - GN MNH Registry data collection forms \\
\hline
\end{tabular}

areas. The primary, secondary and tertiary facilities had 874,2882 , and 34,322 annual deliveries respectively in the pre-HBB period. Their pre-HBB baseline perinatal mortality rates per 1000 births were 13.73, 23.95, and 28.79 respectively. None of the facilities were providing a structured ENC and HBB training prior to the study.

\section{Implementation of HBB training in facilities}

The training for GN-HBB-IS followed the approach developed by the American Academy of Pediatrics and has been described in detail elsewhere [18, 20, 21]. Briefly, it consisted of the training of Master Trainers (MT) who then trained facility birth attendants (BAs) in HBB; the introduction of a multi-faceted monitoring program; and retraining of the active BAs after six months. BAs were instructed to resuscitate all non-macerated births, including those considered fresh stillbirths.

Standard HBB training materials and equipment (Laerdal NeoNatalie ${ }^{\circ}$ equipment and materials and clean delivery kits) were provided to all facilities and MTs based on delivery volume at the time of training - these materials and equipment were not available in the pre-study period. New BA recruits who joined the facility after the initial training were also provided HBB training immediately on recruitment. All the staff of the health facilities were also trained in basic Essential Newborn Care (ENC) [22].

The initial Master Trainers (MT) training workshop was held in May 2012. The Nagpur MT then conducted facility level training workshops for birth attendants (BAs) between June and October 2012. Refresher trainings were conducted six months after the initial HBB training.

\section{Monitoring of HBB implementation in facilities}

Monitoring activities were introduced after the initial facility-level training of BAs and included daily bag and mask ventilation practice sessions when BAs reported for work and signed the logbooks; daily checks of availability, cleanliness, and function of resuscitation equipment; regular observation of deliveries; debriefing after every resuscitation; audits of every perinatal death; once a month quality assurance (QA) visits to observe $\mathrm{HBB}$ skills directly during deliveries or test the BAs using a neonatal simulator if no deliveries were available $[18,20,21]$. Monthly monitoring reports were reviewed and bi-weekly data review calls were made with the central core staff (RTI International and NICHD), followed by feedback to facility MTs and BAs.

\section{Study outcomes for the HBB trained facilities in Nagpur, India}

Our primary hypothesis for the Nagpur-HBB-FS was that here would be at a $20 \%$ decrease in Nagpur facility based PMR-D1 in the 12 months pre vs. post training and implementation period. PMR-D1 included stillbirths or within $24 \mathrm{~h}$ of birth. We explored pre-post differences in stillbirth rates (SBR) and in day-1 neonatal mortality rates (NMR-D1).

Our primary outcome was PMR-D1 pre-post GNHBB-IS. Exploratory outcomes included pre-post GN-HBB-IS SBR (fresh and macerated stillbirths per 1000 total births) and NMR-D1 per 1000 live births. We collected one-year pre training data (April 2011 to March 2012) from the pre-existing standard hospital 
records like birth registers and mortality registers of the Nagpur facilities participating in GN-HBB-IS. The training was conducted from June 2012 to October 2012. The post GN-HBB-IS training data (November 2012 to October 2013) was also collected from similar pre-existing standard hospital records- different from the data collection tools used in the GN-HBB-IS.

In the pre- period, stillbirths were not routinely categorized as fresh or macerated, even though the facilities were trained to make this distinction during the GN-HBB-IS study and in the post period. For this reason, in both periods, we combined fresh and macerated stillbirths and reported them as total stillbirths. Similarly, PMR-D1 was defined as fresh or macerated stillbirths plus deaths within $24 \mathrm{~h}$ of birth. NMR-D1 was defined as neonatal death within $24 \mathrm{~h}$ of a live birth.

\section{Data management}

Primary and secondary outcomes were obtained from the facility medical records by trained data collectors. The following data was collected for pre -post period- number of live births, mode of delivery, presence of multiple gestations, gender of baby and presence of maternal complications (gestational diabetes mellitus, pregnancy induced hypertension, abruptio placentae, eclampsia, sickle cell disease, or other chronic illness) for all PMR-D1.

\section{Power and statistical analysis}

We assumed that the facility based pre GN-HBB-IS PMRD1 would be $25 / 1000$ total births, based on historical data from the facilities. If total births occurring in the year before and after GN-HBB-IS was greater than 19,000 in both periods, the study would have greater than $90 \%$ power to detect a $20 \%$ reduction in pre to post PMR-D1 (Sample size estimates were obtained using nQuery Advisor version 7.0, Statistical Solutions, Boston, 2012).

We compared the primary and exploratory outcomes pre and post the GN-HBB-IS using the Fisher Normal Test. We also used multilevel mixed effects Poisson modeling to predict PMR-D1 based on neonatal and maternal characteristics. The level of care of facility (primary, secondary or tertiary) and time ("pre-HBB period" or "post-HBB period") were fixed effects in the model and the 13 participating facilities were included as random effects to obtain robust standard errors.

\section{Ethical approval}

The study protocol for the Nagpur-HBB-FB study was reviewed and approved by the Institutional Review Board of Lata Medical Research Foundation, Nagpur. All facility-based data was obtained from medical records so no specific consent was obtained from the women giving birth at the facilities.

\section{Results}

As shown in the flow diagram in Fig. 1, there were 78, 948 births in the 13 HBB facilities in Nagpur - 38,078 in the pre-HBB and 40,870 in the post-HBB period. The majority of these births $(71,489)$ took place in tertiary care facilities, 1731 took place in primary and 5728 in secondary facilities. Table 2 shows PMR-D1, SBR and NMR-D1 pre- and post-HBB, all of which were significantly reduced in the post-HBB period. The model yielded a significant incidence risk ratio (IRR) post to pre HBB of 0.85 (95\% CI 0.73-0.98) for PMR-D1, of 0.86 (95\% CI: $0.76-0.98)$ for SBR and of 0.79 (95\% CI: 0.62-1.00) for NMR-D1. Figure 2 shows monthly PMRD1, SBR and NMR-D1 pre- and post- HBB in the 13 facilities, all of which were significantly lower in the post vs. pre HBB period.

Among the PMR-D1, there was no significant difference in the pre vs post period for the baseline maternal

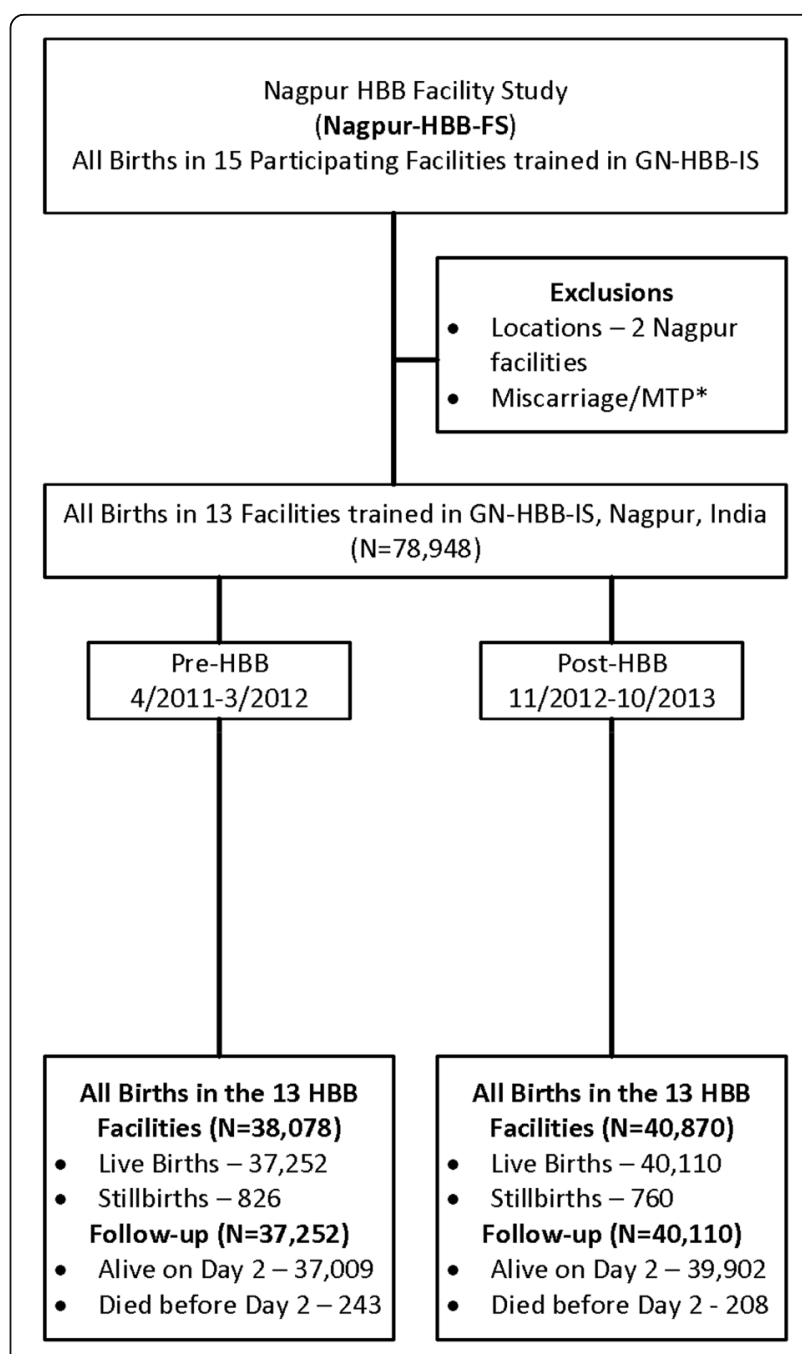

Fig. 1 Flow Diagram for the Nagpur, India HBB Facility Study (Nagpur-HBB-FS) 
Table 2 Nagpur-HBB-FS Outcomes

\begin{tabular}{|c|c|c|c|c|c|}
\hline \multirow[t]{2}{*}{ Outcome } & \multicolumn{2}{|c|}{ Pre HBB } & \multicolumn{2}{|c|}{ Post HBB } & \multirow{2}{*}{$\begin{array}{l}\text { p }[95 \% \mathrm{Cl} \\
\text { for diff.) }\end{array}$} \\
\hline & $n$ & Rate & $n$ & Rate & \\
\hline $\begin{array}{l}\text { 1-Day Perinatal Mortality } \\
\text { (PMR-D1) }\end{array}$ & 1069 & $28.07^{a}$ & 968 & $23.68^{a}$ & $0.0001[2.1,6.6]$ \\
\hline Stillbirths (SB) & 826 & $21.69^{a}$ & 760 & $18.6^{\mathrm{a}}$ & $0.002[1.1,5.0]$ \\
\hline -Day Mortality & 243 & $6.52^{b}$ & 208 & $5.18^{b}$ & $0.006[0.5,3.6]$ \\
\hline
\end{tabular}

$$
\text { (NMR-D1) }
$$

${ }^{a}$ Calculated by total births in the denominator - for pre HBB, $N=38,078$; for post HBB $N=40,870$

${ }^{\mathrm{b}}$ Calculated by live births in the denominator - for pre $\mathrm{HBB}, N=37,252$; for post $\mathrm{HBB} N=40,110$

and neonatal characteristics such as caesarian sections ( $10.8 \%$ vs $12.8 \%)$, prematurity $(66.3 \%$ vs $68.2 \%)$, male gender $(54.5 \%$ vs $53.2 \%)$, multiple gestations $(7.3 \%$ vs $6.0 \%)$ and low birth weight ( $83.3 \%$ vs $86.8 \%)$. Maternal complications were present in $15.3 \%$ in pre and $11.1 \%$ in post period (p: 0.004).

\section{Discussion}

This study showed that by focusing on day 1 perinatal and neonatal mortality just in trained facilities in one of the three GH-HBB-IS sites in Nagpur, India, HBB implementation significantly reduced PMR-D1 by $15.6 \%$, SBR by $14.2 \%$ and NMR-D1 by $20.6 \%$. By contrast, the NICHD GN-HBB-IS did not result in improvements in PMR-D7 in communities that were partially served by the facilities in which staff were trained in the GN-HBBIS in the Nagpur or other GN sites [20]. There could be two likely reasons for this discrepancy. Firstly, there was an overlap of pre-HBB period and the training period in GN-HBB-IS which may have reduced PMRD7 during the pre-period and may have led to the null result. Secondly, the GN-HBB-IS analyzed all births in a community-based registry. Around 45\% of these births did take place in $\mathrm{HBB}$ trained facilities but the rest (more than $50 \%$ of babies) delivered outside in other facilities that were not trained in HBB implementation. Consequently, where the intervention did not happen, there was likely no effect and this diluted the effect in the overall population. In contrast the Nagpur-HBB-FS study analyzes ALL facility births- whether or not they belonged to the MNH registry- from 13 of the 15 facilities that had participated in the GN-HBB-IS. Since around $11 \%$ of these facility births belonged to the $\mathrm{MNH}$ registry, there was about $11 \%$ overlap in the births analyzed in GN-HBB-IS and Nagpur-HBB-FS (See Table 1, Fig. 1, and Additional file 1).

Similar to other studies, we also found a significant reduction in the SBR post $\mathrm{HBB}$ implementation, likely because $\mathrm{HBB}$ improves identification and resuscitation of neonates that appear to be lifeless at birth [23-25].

The main strength of our Nagpur-HBB-FS is that it evaluated the direct impact of $\mathrm{HBB}$ implementation (HBB Training, equipment's, and monitoring activities as explained in methods) with in a range of primary care facilities (where birth attendants may have minimal training) to tertiary care facilities (with high patient volumes and likely more complicated deliveries). Important limitations include lack of information on whether the study population changed in the pre to post period and the impact of $\mathrm{HBB}$ training on how perinatal mortality and stillbirths were recorded in the medical record in the two periods. Also, since many newborns get discharged from facilities before 7 days, in absence of any follow up information on the newborn's status at home, we were unable to report PMR-D7 in the Nagpur-HBBFS to assess whether the improvements seen in PMR-D1 were sustained through day 7 .

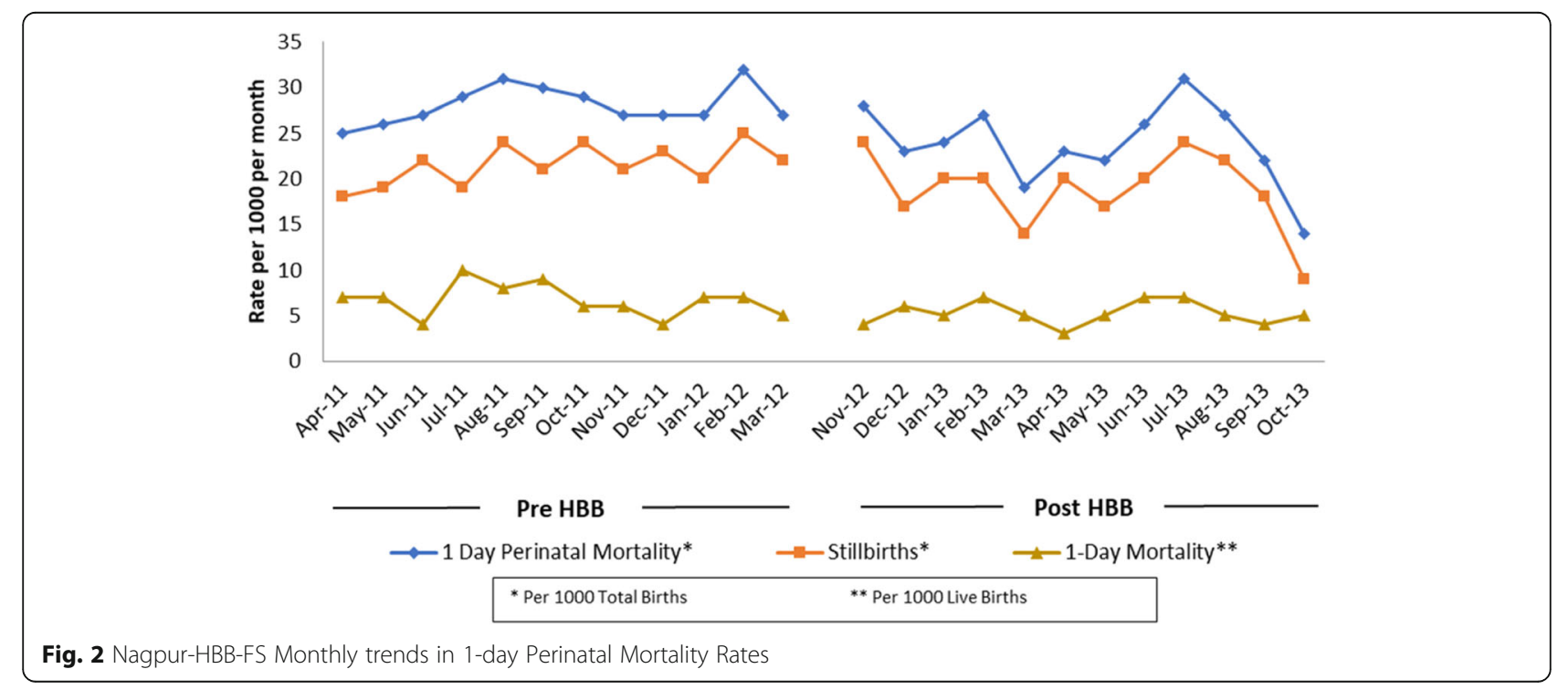


So, where does this analysis fit in our understanding of the role of HBB implementation in neonatal resuscitation? Our study suggests that HBB improves PMR-D1 but may not translate to subsequent neonatal survival as the impact of neonatal resuscitation trainings on reduction of neonatal deaths will be highest for 1-day deaths and diminish subsequently. This fact has been endorsed in a recent meta-analysis of 20 studies that assessed impact of neonatal resuscitation trainings which showed a $42 \%$ reduction in 1day mortality and 18\% reduction in 7-day mortality [26]. Reduction of 28-days neonatal mortality will thus require, in addition to resuscitation trainings, provision of specialized care to the resuscitation survivors and various other existing intervention packages like improved hygiene at birth, breastfeeding and simple approaches to keep babies warm etc. $[27,28]$ as predominant causes of mortality after $24 \mathrm{~h}$ of life are prematurity, hypoxic ischemic encephalopathy, sepsis and congenital malformations $[29,30]$. The GN-HBB-IS provides a bold target for where we need to go to improve global neonatal survival. Focus on facilities alone may not be sufficient to translate the gains achieved in neonatal survival to be generalized to population level gains.

\section{Conclusion}

This study addresses differences between facility based perinatal mortality rates on day 1 of life and community based perinatal mortality rates through day 7 of life. In this study (Nagpur-HBB-FS), facility based SBR, NMR-D1, and PMR-D1 were significantly lower after HBB implementation. In the previously published study (GN-HBB-IS), these facility based benefits did not translate to improvements in PMR-D7 in communities served by these facilities, possibly because all the community births did not occur in the facilities where HBB was implemented or because additional interventions are needed after day 1 of life. Benefits of HBB may thus be limited if births do not occur in facilities where HBB training has been provided. Increased access to facilities with HBB training will reduce perinatal mortality in that community.

\section{Additional file}

Additional file 1: Flow Diagram for the Nagpur Site of the Global Network HBB Implementation Study. (DOCX 72 kb)

\footnotetext{
Abbreviations

BAs: Birth Attendants; ENC: Essential Newborn Care; GN: Global Network for Women's and Children's Health Research; GN-HBB-IS: Global Network Helping Babies Breathe Implementation Study; HBB: Helping Babies Breathe; MT: Master Trainer; MTP: Medical Termination of Pregnancy; Nagpur-HBB-FS: Nagpur Helping Babies Breathe Facility Study; NICHD: The Eunice Kennedy Shriver National Institute of Child Health and Human Development; NMR: Neonatal Mortality Rate; NRP: Neonatal Resuscitation Program; PMR: Perinatal Mortality Rate; RTI: Research Triangle Institute; SBR: Stillbirth Rate
}

\section{Acknowledgements}

We thank the Eunice Kennedy Shriver National Institute of Child Health and Human Development's Global Network for Women's and Children's Health Research, Norad and Laerdal Foundation for funding the GN HBB IS. We thank all HBB Master Trainers and Facilitators and BAs from the participating facilities who made the HBB implementation possible.

\section{Authors' contributions}

This concept was proposed, designed and overseen at Nagpur, India site by $\mathrm{AP}$ and $\mathrm{AB}$ in collaboration with PLH. All authors have taken part in the overall execution of the study and in writing the draft report. Data collection, data cleaning (edit rectification) were done by SB, KK and SA. Overall monitoring and supervision of data collection was done by AP. AAP performed statistical analysis in collaboration with AP. All authors approved the final manuscript.

\section{Funding}

The data collection, design, analysis and manuscript of this study (Nagpur-HBB-FS) were funded by Lata Medical Research Foundation, Nagpur, India. The HBB Training was a part of the GN-HBB-IS which was funded by the Eunice Kennedy Shriver National Institute of Child Health and Human Development's Global Network for Women's and Children's Health Research, NORAD and Laerdal Foundation.

\section{Availability of data and materials}

A de-identified minimal dataset for the Nagpur-HBB-FS can be shared upon reasonable request to the corresponding author. All authors hereby declare that individual participant data, in any form is not a part of this publication.

\section{Ethics approval and consent to participate}

The study protocol for the Nagpur-HBB-FS was reviewed and approved by the Institutional Review Board of Lata Medical Research Foundation, Nagpur. Written informed consent for de-identified hospital data abstraction of stillbirths and day 1 neonatal deaths was obtained from the head of that department. The ethical approvals for the previously published GN-HBB-IS that trained the Nagpur facilities in HBB have been published previously $[18,20,21]$.

\section{Consent for publication}

Not applicable.

\section{Competing interests}

The authors declare that they have no competing interests.

\section{Author details}

${ }^{1}$ Lata Medical Research Foundation, Nagpur, India. ${ }^{2}$ Mahatma Gandhi Institute of Medical Sciences, Sewagram, Maharashtra State 442102, India.

${ }^{3}$ Ankura Hospital for Women and Children, Hyderabad, India. ${ }^{4}$ Boston University School of Public Health, Boston, MA, USA.

Received: 16 January 2019 Accepted: 27 August 2019

Published online: 04 September 2019

\section{References}

1. Hug L, Alexander M, You D, et al. National, regional, and global levels and trends in neonatal mortality between 1990 and 2017, with scenario-based projections to 2030: a systematic analysis. Lancet Glob Health. 2019;7:e71020. https://doi.org/10.1016/S2214-109X(19)30163-9.

2. Lawn JE, Blencowe $H$, Oza $S$, et al. Every newborn: progress, priorities, and potential beyond survival. Lancet. 2014;384:189-205. https://doi.org/10.1016/ S0140-6736(14)60496-7.

3. Oestergaard MZ, Inoue M, Yoshida S, et al. Neonatal mortality levels for 193 countries in 2009 with trends since 1990: a systematic analysis of progress, projections, and priorities. PLoS Med. 2011;8:e1001080. https://doi.org/10.13 71/journal.pmed.1001080.

4. Rajaratnam JK, Marcus JR, Flaxman AD, et al. Neonatal, postneonatal, childhood, and under-5 mortality for 187 countries, 1970-2010: a systematic analysis of progress towards Millennium Development Goal 4. Lancet. 2010; 375:1988-2008. https://doi.org/10.1016/S0140-6736(10)60703-9.

5. Goudar SS, Carlo WA, McClure EM, et al. The maternal and newborn health registry study of the global network for Women's and Children's Health 
Research. Int J Gynaecol Obstet. 2012;118:190-3. https://doi.org/10.1016/j. ijgo.2012.04.022.

6. Lawn JE, Blencowe H, Waiswa P, et al. Stillbirths: rates, risk factors, and acceleration towards 2030. Lancet Lond Engl. 2016;387:587-603. https://doi. org/10.1016/S0140-6736(15)00837-5

7. Dilenge M-E, Majnemer A, Shevell MI. Topical review: long-term developmental outcome of asphyxiated term neonates. J Child Neurol. 2001;16:781-92. https://doi.org/10.1177/08830738010160110201.

8. Birth asphyxia: A major killer of newborns. POPLINE.org Published Online First: 2006.https://www.popline.org/node/198594 (accessed 2 Jul 2018).

9. Palme-Kilander C. Methods of resuscitation in low-Apgar-score newborn infants--a national survey. Acta Paediatr. 1992;81:739-44.

10. Kattwinkel J, Niermeyer S, Nadkarni V, et al. Resuscitation of the newly born infant: an advisory statement from the pediatric working Group of the International Liaison Committee on resuscitation. Resuscitation. 1999;40:71-88.

11. Wyckoff MH, Aziz K, Escobedo MB, et al. Part 13: neonatal resuscitation: 2015 American Heart Association guidelines update for cardiopulmonary resuscitation and emergency cardiovascular care. Circulation. 2015;132: S543-60. https://doi.org/10.1161/CIR.0000000000000267.

12. Trevisanuto D, Bertuola F, Lanzoni P, et al. Effect of a neonatal resuscitation course on healthcare providers' performances assessed by video recording in a low-resource setting. PLoS One. 2015;10. https://doi.org/10.1371/journal. pone.0144443.

13. Wall SN, Lee ACC, Niermeyer S, et al. Neonatal resuscitation in low-resource settings: what, who, and how to overcome challenges to scale up? Int J Gynaecol Obstet. 2009;107(Suppl 1):S47-62-63-64. https://doi.org/10.1016/j. ijgo.2009.07.013.

14. WHO | Basic newborn resuscitation: a practical guide. WHO. 2018.http:// www.who.int/maternal_child_adolescent/documents/who_rht_msm_981/ en/ (accessed 24 Aug 2018).

15. Zhu XY, Fang $H Q$, Zeng $S P$, et al. The impact of the neonatal resuscitation program guidelines (NRPG) on the neonatal mortality in a hospital in Zhuhai, China. Singap Med J. 1997;38:485-7.

16. Hofmeyr GJ, Haws RA, Bergström S, et al. Obstetric care in low-resource settings: what, who, and how to overcome challenges to scale up? Int J Gynaecol Obstet. 2009;107 Suppl 1:S21-44, S44-45. https://doi.org/10.1 016/j.ijgo.2009.07.017.

17. Lawn JE, Lee ACC, Kinney M, et al. Two million intrapartum-related stillbirths and neonatal deaths: where, why, and what can be done? Int J Gynaecol Obstet. 2009;107(Suppl 1):S5-18, S19. https://doi.org/10.1016/ j.ijgo.2009.07.016.

18. Bang A, Bellad R, Gisore P, et al. Implementation and evaluation of the helping babies breathe curriculum in three resource limited settings: does helping babies breathe save lives? A study protocol. BMC Pregnancy Childbirth. 2014;14:116. https://doi.org/10.1186/1471-2393-14-116.

19. Bang AT, Bang RA, Baitule SB, et al. Management of birth asphyxia in home deliveries in rural Gadchiroli: the effect of two types of birth attendants and of resuscitating with mouth-to-mouth, tube-mask or bag-mask. J Perinatol. 2005;25(Suppl 1):S82-91. https://doi.org/10.1038/sj.jp.7211275.

20. Bellad RM, Bang A, Carlo WA, et al. A pre-post study of a multi-country scale up of resuscitation training of facility birth attendants: does helping babies breathe training save lives? BMC Pregnancy Childbirth. 2016;16:222. https:// doi.org/10.1186/s12884-016-0997-6.

21. Bang A, Patel A, Bellad R, et al. Helping babies breathe (HBB) training: what happens to knowledge and skills over time? BMC Pregnancy Childbirth. 2016;16:364. https://doi.org/10.1186/s12884-016-1141-3.

22. WHO | Essential newborn care course. http://www.who.int/maternal_child_ adolescent/documents/newborncare_course/en/ (accessed 4 Jul 2018).

23. Goudar SS, Somannavar MS, Clark R, et al. Stillbirth and newborn mortality in India after helping babies breathe training. Pediatrics. 2013;131:e344-52. https://doi.org/10.1542/peds.2012-2112.

24. Msemo G, Massawe A, Mmbando D, et al. Newborn mortality and fresh stillbirth rates in Tanzania after helping babies breathe training. Pediatrics. 2013;131:e353-60

25. Carlo WA, Goudar SS, Jehan I, et al. Newborn-care training and perinatal mortality in developing countries. N Engl J Med. 2010;362:614-23. https:// doi.org/10.1056/NEJMsa0806033.

26. Patel A, Khatib MN, Kurhe $K$, et al. Impact of neonatal resuscitation trainings on neonatal and perinatal mortality: a systematic review and meta-analysis. BMJ Paediatr Open. 2017;1:e000183. https://doi.org/10.1136/bmjpo-2017-000183.
27. Darmstadt GL, Bhutta ZA, Cousens S, et al. Evidence-based, cost-effective interventions: how many newborn babies can we save? Lancet. 2005;365: 977-88. https://doi.org/10.1016/S0140-6736(05)71088-6.

28. Bhutta ZA, Darmstadt GL, Hasan BS, et al. Community-based interventions for improving perinatal and neonatal health outcomes in developing countries: a review of the evidence. Pediatrics. 2005;115:519-617. https://doi. org/10.1542/peds.2004-1441.

29. Mwaniki MK, Atieno M, Lawn JE, et al. Long-term neurodevelopmental outcomes after intrauterine and neonatal insults: a systematic review. Lancet. 2012;379:445-52. https://doi.org/10.1016/50140-6736(11)61577-8.

30. Jacob J, Kamitsuka M, Clark RH, et al. Etiologies of NICU deaths. Pediatrics. 2015;135:e59-65. https://doi.org/10.1542/peds.2014-2967.

\section{Publisher's Note}

Springer Nature remains neutral with regard to jurisdictional claims in published maps and institutional affiliations.
Ready to submit your research? Choose BMC and benefit from:

- fast, convenient online submission

- thorough peer review by experienced researchers in your field

- rapid publication on acceptance

- support for research data, including large and complex data types

- gold Open Access which fosters wider collaboration and increased citations

- maximum visibility for your research: over $100 \mathrm{M}$ website views per year

At BMC, research is always in progress.

Learn more biomedcentral.com/submissions 